\title{
Association Between Nonalcoholic Fatty Liver Disease and the Incidence of Asthma in Adults: Finding from a Nationwide Study in Korea
}

\author{
Jae-Hyung Roh \\ Chungnam National University \\ Hanbyul Lee \\ Kyungpook National University \\ Yun-Jeong Bae \\ Asan Medical Center \\ Chan Sun Park \\ Inje University Haeundae Paik Hospital \\ Hyo-Jung Kim \\ Inje University Haeundae Paik Hospital \\ Sun-Young Yoon ( $\sim$ ggulcha2000@naver.com) \\ Chungnam National University
}

\section{Research Article}

Keywords: Asthma, fatty liver index, incidence, metabolic syndrome, nonalcoholic fatty liver disease

Posted Date: June 14th, 2021

DOI: https://doi.org/10.21203/rs.3.rs-579281/v1

License: (1) (1) This work is licensed under a Creative Commons Attribution 4.0 International License. Read Full License 


\section{Abstract}

Asthma and nonalcoholic fatty liver disease (NAFLD) are chronic diseases and are known to be associated with metabolic abnormalities. We aimed to clarify the association between NAFLD and the incidence of asthma in a large population-based cohort. We selected 160,603 healthy individuals without comorbidities from the National Health Insurance Service-National Sample cohort during 2009-2014. . NAFLD was defined by a surrogate marker, the fatty liver index (FLI). During a median of 5.08 years' follow-up, 16,377 subjects (10.2\%) were newly diagnosed with asthma and categorized into three groups according to FLI. The cumulative incidence of asthma was higher in subjects with higher vs. lower FLIs (FLI $<3010.1 \%, 30 \leq \mathrm{FLI}<6010.8 \%, \mathrm{FLI} \geq 6010.5 \%)$. Higher FLI associated with an increased incidence of asthma (Harzard ratios (HR)highest vs. lowest FLI, 1.25; $95 \% \mathrm{Cl}, 1.15-1.36$ ). The results using another definition of NAFLD as measured by hepatic steatosis index (HIS) are similar to the primary results. This association was more pronounced in women than in men (HR 1.46; $95 \% \mathrm{Cl}, 1.13-1.64$ vs. HR $1.07 ; 95 \% \mathrm{Cl}, 0.94-1.20)$. This study demonstrated that NAFLD as measured by FLI and HIS may influence the incidence rates of asthma in adults, especially in women.

\section{Introduction}

Nonalcoholic fatty liver disease (NAFLD) is the most common chronic liver disease worldwide, and its prevalence in Asian-Pacific countries is estimated at $20-30 \%{ }^{1,2}$.

NAFLD is associated with an increase of multisystem diseases including diabetes, ischemic cardiovascular disease, and chronic kidney disease $^{3,4}$. Recently, an association of NAFLD with atrial fibrillation, a non-ischemic cardiac disease, has also been reported ${ }^{5}$.

Asthma is the most common chronic airway disease, with approximately 350 million cases reported worldwide in $2015^{6}$. Recent studies have reported the epidemiological link between metabolic syndrome (MetS) and asthma, especially obesity is a well-known risk factor for asthma and may mediate its severity ${ }^{7,8}$. Several studies have reported the association of asthma with other components of MetS, such as

dyslipidemia and insulin resistance, as well as obesity ${ }^{9-11}$. NAFLD and MetS also share many features, and there is growing evidence of a bidirectional relationship between the two diseases ${ }^{12,13}$. However, to date, no studies have investigated the direct association between NAFLD and the development of asthma.

We hypothesized that NAFLD is associated with the incidence of asthma. The objective of this study was to investigate the direct association between NAFLD and asthma incidence using a large, claim-based cohort consisting of healthy Korean adult population without traditional risk factors and comorbidities.

\section{Methods}

\section{Database and study cohort}

We conducted a retrospective population-based cohort study using data from the National Health Insurance Service-National Sample Cohort 2.0 (NHIS-NSC 2.0) 2002-2015, as previously reported ${ }^{14,15}$. This database contains data collected from cohort members on sociodemographic characteristics, all medical claims including diagnosis, medical treatment, and health care utilization; and is linked with their data from the National Health Screening database. The database also includes mortality data such as the date and cause of death based on the death registration database of Statistics Korea. This study was approved by the Institutional Review Board of Chungnam National University Hospital, Sejong, Korea (protocol no. 2020-09-023). Witten consents were waived by ethics committee due to the retrospective nature of the study.

\section{Study subjects}

All subjects aged 20 years and older who underwent national health screening at least once between January 2009 and December 2014 were enrolled in this study. The first health check-up was considered as the index check-up, and the year of the index check-up was the index year. Subjects who met the following exclusion criteria were excluded from the analysis: i) diagnosed with asthma within two years before the index year; ii) diagnosed with comorbid conditions including other pulmonary disease, liver disease, heart failure, hypertension, diabetes, cerebrovascular disease, ischemic heart disease, valvular heart disease, or peripheral artery disease within two years before the index year; iii) had a prescription of oral hypoglycemic, antihypertensive, or lipid-lowering agents within two years before the index year; iv) had any smoking history (current or former smoker); and v) had missing data in the index check-up and follow-up period. 
National health screenings included physical examination, medical history, blood test, and chest X-ray, and collection of data on healthrelated behavior such as exercise habits, smoking, and alcohol consumption using self-administered questionnaires. Each diagnosis was defined using the $10^{\text {th }}$ revision of the International Classification of Diseases (ICD-10). Each diagnosis code used in the analysis is shown in a supplemental table (Table S1).

\section{Definition of adult-onset asthma cases and asthma-related healthcare use}

The definition of asthma was as follows: i) Individuals with at least two or more claims with asthma according to the ICD-10 code (J45.XJ46.X) for the primary or sub-diagnosis; ii) prescribed at least one of the asthma-related medications: inhaled corticosteroids (ICS), ICS and LABA combined into a single inhaler (ICSs/LABAs), systemic LABAs, oral leukotriene antagonists (LTRA), short-acting $\beta$ 2-agonists (SABAs), xanthine derivatives, and systemic corticosteroids ${ }^{16}$. In terms of asthma-related healthcare use, outpatient visits were defined as patients diagnosed with asthma related ICD-10 codes (J45-46) and prescribed at least one of the asthma-related medications (see the above). Asthma-related hospitalization or emergency department (ED) visits were defined as admission and ED visit under asthma related ICD-10 codes and prescribed oral or injected systemic corticosteroids to exclude healthcare use for other principal purposes ${ }^{15,17}$.

\section{Data collection and measure}

Body mass index (BMI) was calculated as weight $(\mathrm{kg})$ divided by the square of the height $(\mathrm{m})$. Obesity was defined as a BMI of $\geq 25 \mathrm{~kg} / \mathrm{m}^{2}$ according to the World Health Organization criteria for Asian populations ${ }^{18}$. Alcohol consumption and daily activity were measured using a self-reported questionnaire.

Fatty liver index (FLI) is a noninvasive surrogate marker of NAFLD and is reported to be reliable in previous epidemiological studies ${ }^{19-22}$. FLI consists of BMI, waist circumference, triglyceride, and gamma-glutamyltransferase (GGT) and is calculated using the following formula:

$\mathrm{FLI}=\frac{e^{0.953 \ln (T G)+0.139 \times B M I+0.713 \times \ln (G G T)+0.053 \times W C-15.745}}{1+e^{0.953 \ln (T G)+0.139 \times B M I+0.718 \times \ln (G G T)+0.053 \times W C-15.745}} \times 0.0$

The FLI score ranges from 0 to 100, and the original study suggested an FLI score of $\geq 60$ as the cut-off value to diagnose hepatic steatosis. In this study, subjects were then categorized into three groups: $0 \leq \mathrm{FLI}<30,30 \leq \mathrm{FLI}<60$ and FLI $\geq 60$, based on previous studies $^{21}$.

However, few studies have been conducted to validate this application of FLI in Asian populations, taking into consideration ethnic characteristics such as lower BMI and waist circumference compared to those of Western populations. We therefore conducted supplemental analysis using different FLI cut-off criteria: $0 \leq \mathrm{FLI}<25,25 \leq \mathrm{FLI}<35$ and FLI $\geq 35$ for males and $0 \leq \mathrm{FLI}<10,10 \leq \mathrm{FLI}<$ 20 and $\mathrm{FLI} \geq 20$ for females, proposed by Yang et al in a study conducted among Taiwanese people ${ }^{22}$. Other surrogate marker of fatty liver, hepatic steatosis index (HIS) was also calculated as supplementary criteria. HIS consists of liver enzymes, such as alanine transaminase (ALT), aspartate aminotransferase (AST), and BMI, calculated using the following formula: HIS = 8x (ALT/ AST ratio) + BMI $\left(+2\right.$, if female; +2 , if diabetes mellitus). HIS values of $\geq 36$ rule in hepatic steatosis ${ }^{23}$.

\section{Statistical analysis}

Data are presented as mean \pm standard deviation for continuous variables and number (percentage) for categorical variables. One-way analysis of variance and chi-square tests were used to compare the differences between the FLI quartile groups. The incidence rate of asthma was expressed as the total number of newly diagnosed asthma cases per 100,000 person-years. Cumulative incidence rates were calculated and compared between quartile-based groups using Kaplan-Meier estimates and log-rank tests. Cox proportional hazards regression was used to estimate adjusted hazard ratios (HRs) and 95\% confidence intervals (Cl) for asthma incidence. Covariates included in Model 1 were age and sex. Later, clinical characteristics of variables whose association with adult-onset asthma showed borderline statistical significance $(P<.10)$, physical activity, alcohol drinking, systolic blood pressure, diastolic blood pressure, fast blood glucose and low density lipoprotein cholesterol were incorporated into the existing Model 1 to obtain Model 2. During subgroup analyses, FLI was integrated into the statistical models as a continuous variable after log transformation. A two-sided $P$ value of $<0.05$ was considered to indicate statistical significance. Statistical analyses were performed using R software, version 3.3.3 (R Foundation for Statistical Computing, Vienna, Austria; www.r-project.org).

\section{Results}




\section{Baseline characteristics}

Of the total 556,884 NHIS-NSC 2.0 sample cohort population, the data of 160,603 individuals were analyzed after excluding subjects who met the exclusion criteria (Figure 1). The study subjects were divided into three groups according to their FLI value $(0 \leq \mathrm{FLI}<30,30 \leq \mathrm{FLI}$ $<60$ and $\mathrm{FLI} \geq 60$ ). Detailed baseline characteristics by FLI groups are shown in Table 1 . Groups with higher FLI had older individuals and a greater proportion of obese and male subjects than groups with lower FLI. Mean blood pressure and the proportion of alcohol consumers also tended to increase with FLI. In terms of laboratory data, the lipid profile progressively worsened with increasing FLI value, and GGT and fasting glucose levels were also higher in patients with higher FLI.

\section{Relationship between FLI and the incidence of asthma}

During 5.08 years' median follow-up (interquartile range, 3.08-6.16), a total of 16,377 subjects (10.2\%) developed asthma. The incidence of asthma was high in groups with higher FLI (FLI < 30 10.1\% [13,744/136,094], $30 \leq \mathrm{FLI}<6010.8 \%[1,986 / 18,373], \mathrm{FLI} \geq 6010.5 \%$ $[647 / 6,136])$; the cumulative incidence of asthma is shown in Figure 2.

Table 2 shows hazard ratios and their $95 \% \mathrm{Cl}$ from Cox proportional hazards regression analysis for subjects with adult-onset asthma according to the FLI group. After adjusting for age and sex (Model 1), the HR (95\% Cl) was 1.23 (1.13-1.33) for the highest group of FLI compared with that of the lowest. When the model was further adjusted for physical activity, alcohol drinking, systolic blood pressure, diastolic blood pressure, fast blood glucose, and low-density lipoprotein cholesterol (Model 2), the HR $(95 \% \mathrm{Cl})$ was 1.25 (1.15-1.36) for the highest group of FLI compared with that of the lowest. In addition to the FLI groups, the analysis conducted after categorizing the study subjects according to the different FLI cut-off criteria suggested in previous studies (HR 1.18,95\% Cl 1.14-1.22) and $\mathrm{HIS}$ ( $\mathrm{HR} 1.12,95 \% \mathrm{Cl}$ 1.07-1.17), showed that asthma incidence tended to increase as each value increased (Table 2).

Furthermore, we performed subgroup analysis the association between asthma incidence and FLI according to sex. As a result, the FLI was associated with asthma incidence for both sexes, but was more pronounced in women (HR 1.46, 95\% Cl, 1.13-1.64 vs. HR 1.07, 95\% Cl, 0.94-1.20, Table 3).

\section{Utilization of health care services}

During the follow-up period, the highest FLI group had a lower asthma-related hospitalization rate compared to the rest of the groups $(0 \leq \mathrm{FLI}<30,33.5 \% ; 30 \leq \mathrm{FLI}<60,35.1 \% ; \mathrm{FLI} \geq 60,29.5 \%, P<0.001)$. However, the average frequency (2.08 vs. $2.54, P<0.001)$, and days (8.18 vs. 19.78 per person; 8.72 vs. 11.54 per admission) were significantly higher in the group with high FLI (Table 3). Asthma-related intensive care unit transfer tended to increase with increasing $\mathrm{FLI}(0.04 \%-0.08 \%, P<0.001)$, and there were significantly more outpatient visits in the higher FLI group $(59.7 \%$ vs. $63.4 \%, P<0.001)$.

\section{Subgroup analysis of clinical variable affecting asthma incidence}

The incidence of asthma in each clinical subgroup is shown in Figure S1. In all subgroups except alcohol drinkers, asthma occurrence tended to increase as FLI increased.

Table S3 presents the hazard ratios for asthma incidence according to each clinical variable. As the age and BMI of the study population increased, the HR tended to increase slightly, and the incidence of asthma was higher in women than in men (male, $\mathrm{HR} \mathrm{0.69,95 \%} \mathrm{Cl}, 0.67$ 0.72). Total cholesterol and low-density lipoprotein cholesterol were also associated with increased HR. Daily physical activity had a protective effect against asthma incidence. It was observed that the difference in asthma incidence according to sex was very distinct, we performed subgroup analysis the association between asthma incidence and FLI according to sex. As a result, the FLI was associated with asthma incidence for both sexes, but the difference of $\mathrm{HR}$ was observed in men (HR 1.07,95\% Cl, 0.94-1.20) and women ( $\mathrm{HR} 1.46,95 \% \mathrm{Cl}$, 1.13-1.64, Table S4).

\section{Discussion}

In this study, we investigated the association between NAFLD as measured using FLI, a validated surrogate marker, and the incidence of asthma in a large, representative sample of the Korean population. NAFLD was associated with an increase in the incidence of asthma and in most clinical subgroups except alcohol drinkers, an increase in the incidence of asthma was observed with an increase in the FLI score. Furthermore, as the FLI score increased, the utilization of asthma-related health care services such as hospitalization days and outpatient visits also increased. 
In current study, the incidence of asthma was slightly higher in the middle group $(30 \leq \mathrm{FLI}<60)$ compared to the highest FLI group. However, as a result of confirming the hazard ratios, it was found that asthma incidence increased as FLI increased, and the same result was obtained in the analysis using other types of FLI categories and HIS, another surrogate marker of NAFLD. Therefore, our findings support the possibility of NAFLD influencing the occurrence of asthma.

NAFLD is highly prevalent worldwide, and it is known that NAFLD occurs along the lines of MetS progression ${ }^{24}$. Similar to MetS, the association of NAFLD with extrahepatic complications such as cardiovascular disease, chronic kidney disease and insulin resistance are well known ${ }^{3,4,24}$. Because potential associations with other diseases including atrial fibrillation and periodontitis have recently been suggested, there is increased interest in the effect of NAFLD on multiple organs 5,25 .

Numerous studies have reported a correlation between MetS and impairment of lung function. Of the MetS components, robust epidemiological data linking abdominal obesity with the incidence and exacerbation of asthma are available ${ }^{26}$. Other factors such as dyslipidemia, hyperglycemia, and hypertension have also shown to be independently associated with asthma. Waist circumference and insulin resistance were revealed independent risk factors for asthma in a large Norwegian adult asthma cohort, and high triglyceride (TG) level and insulin resistance were independently associated with asthma incidence in a cross-sectional study of children aged 4-12 years in West Virginia ${ }^{9,27}$.

Given the above results, a relationship between asthma and NAFLD may be suspected. Therefore, we evaluated the association between these two diseases using a large population after excluding known risk factors such as diabetes, hyperlipidemia, hypertension, and smoking exposure and confirmed the independent association of NAFLD with asthma incidence. To the best of our knowledge, this is the first study to confirm the association between asthma incidence and NAFLD. However, the magnitude of association was not very high, and the following explanation of our results may be considered.

Because the study used health insurance data, asthma patients of all severity levels were included because it was not possible to determine the control status or severity level. Given that less than $10 \%$ of all asthma patients were diagnosed with severe asthma, it is believed that a number of mild asthmatic patients were included, which could have diluted the association.

The association between NAFLD and asthma incidence identified in this study may be explained by several pathophysiological mechanisms underlying the two diseases. First, in patients with NAFLD, elevated levels of circulatory TG and free fatty acids have been observed. Excess fat increases the production of proinflammatory cytokines such as tumor necrosis factor-alpha and interleukin- 6 through the activation of innate immune responses such as pattern recognition receptor ${ }^{28,29}$. Excess fatty acid is also involved in the production of reactive oxygen species by inducing endoplasmic reticulum stress ${ }^{30}$. Increased proinflammatory cytokines and oxidative stress may cause airway epithelial damage and airway inflammation, which may affect asthma development ${ }^{31,32}$. Second, insulin resistance, which plays a key role in the development of fatty liver disease, leads to excessive insulin secretion in the blood. Insulin is thought to contribute to airway hypersensitivity and airway remodeling by causing epithelial damage and smooth muscle proliferation in the airway ${ }^{33}$. In addition, hyperinsulinemia was reported to have been involved in enhancing airway responsiveness through parasympathetic stimulation in an asthma mouse model ${ }^{34}$.

Similar to the above-mentioned observations, our study also confirmed that an increase in age, weight, or poor lipid profile affects the incidence of asthma. Daily physical activity tended to have a positive effect on the suppression of asthma.

Furthermore, we analyzed the correlation between FLI and asthma-related healthcare service utilization, and the number of patients who experienced hospitalization was higher in the group with low FLI, but the number of hospitalizations per patient, number of days of hospitalization, and outpatient frequency increased with higher FLI. The discrepancy between the incidence of asthma and asthma-related hospitalization may have been influenced by the fact that the severity of asthma was not reflected in this study. In fact, the increase in the number of hospitalizations per patient, the number of days of hospitalization, and intensive care unit admission in the high FLI group in this study may also suggest a possible association between FLI and asthma severity. In a study of more than 70,000 adolescents in Brazil published by Kuchinir et al., a significant association was reported between MetS and asthma control ${ }^{35}$. In a similar vein, progression of NAFLD may also affect the severity of asthma. Further research is needed that reflects the severity of asthma.

As another important point, in this study, women showed a stronger correlation between FLI and asthma incidence than men. Several previous studies reported a higher correlation between obesity and asthma in women ${ }^{36,37}$, but others provided conflicting results ${ }^{38}$. Some studies have reported the correlation between metabolic abnormality and the occurrence of asthma ${ }^{9,39}$, but few studies have investigated gender differences. There are several possible mechanisms for differences in the association between asthma incidence and FLI by

Page 5/14 
gender. It is thought that the female sex hormone estrogen may be involved in Th2 inflammation and airway hypersensitivity ${ }^{40}$. It has also been suggested that the level of leptin, an adipokine with pro-inflammatory effect, is high in women independently of body fat mass ${ }^{41}$. Overlapping genetic foci associated with asthma and obesity have been reported, and genetic differences are thought to have an effect ${ }^{42,43}$, but it is still unclear.

Currently, NAFLD is a disease of increasing global concern, several guidelines recommend regular screening in patients with chronic conditions such as obesity, diabetes mellitus, and MetS ${ }^{44}$. In addition to weight reduction, oral hypoglycemic agents are currently being used in an attempt to treat NAFLD ${ }^{44}$. Based on the results of the current study, improvement of NAFLD through such interventions is expected to have a positive effect on asthma. Further studies are needed in the future.

This study has some limitations. First, the incidence of asthma may be overestimated because asthma patients may asymptomatic or have symptoms but do not need medical care for years. To minimize this error, all patients who were diagnosed with asthma or were prescribed asthma-related medication within two year before the index year were excluded based on previous studies ${ }^{45}$. However, the duration of two year may not have been sufficient. However, since the focus of current study was not on the asthma incidence rate itself, but on the identification of the correlation between asthma incidence and fatty liver, it seems that the effect on the outcome was not significant. Second, in this study, determination of NAFLD status did not involve imaging tests such as abdominal ultrasonography or magnetic resonance imaging, which are currently first-line diagnostic methods. However, the FLI used in the current study was validated as a predictor of NAFLD in several existing studies in both Asian and Western populations ${ }^{19,21,22,46}$. In addition, health checkups in various countries currently do not include imaging tests. Therefore, we think that FLI, which can be easily obtained from data obtained from health checkups, may rather comprehensively reflect the presence of hepatic steatosis in the general population. Third, we were unable to include family history of allergic disease, allergen exposure, and atopic status known as asthma risk factors in this study due to the nature of health insurance data. Finally, the generalizability of our study findings is limited to the Korean population. Further studies will be needed for other ethnic groups.

In conclusion, the results of this study suggest that NAFLD, which was measured using the FLI, was associated with an increase in asthma incidence in healthy Korean population, especially in women. It was also confirmed that progression of NAFLD may affect the severity of asthma. These findings suggest that clinicians should be aware of the higher risk of asthma among patients with NAFLD. Further research on the impact of NAFLD management on the incidence and severity of asthma is needed.

\section{Declarations}

\section{Acknowledgements}

This work was supported by research fund of Chungnam National University

\section{Author contributions}

JHR, HBL, YJB and SYY were involved in investigation. HJK, YJB and CSP gave constructive criticism of the study manuscript. JHR, HBL, HJK, CSP and SYY were involved on study conceptualization and interpretation. HBL was involved in data collection. JHR, HBL and SYY contributed to the statistical analysis. JHR and SYY wrote the manuscript with input from all authors.

\section{Competing interests}

The authors have no conflicts of interest to disclosure

\section{References}

1. Younossi, Z. M. et al. Global epidemiology of nonalcoholic fatty liver disease-Meta-analytic assessment of prevalence, incidence, and outcomes. Hepatology 64, 73-84, doi:10.1002/hep.28431 (2016).

2. Li, Z. et al. Prevalence of nonalcoholic fatty liver disease in mainland of China: a meta-analysis of published studies. $J$ Gastroenterol Hepatol 29, 42-51, doi:10.1111/jgh.12428 (2014).

3. Targher, G. et al. Nonalcoholic fatty liver disease and risk of future cardiovascular events among type 2 diabetic patients. Diabetes $\mathbf{5 4}$, 3541-3546, doi:10.2337/diabetes.54.12.3541 (2005).

4. Targher, G. et al. Non-alcoholic fatty liver disease is independently associated with an increased prevalence of chronic kidney disease and proliferative/laser-treated retinopathy in type 2 diabetic patients. Diabetologia 51, 444-450, doi:10.1007/s00125-007-0897-4 
(2008).

5. Roh, J. H. et al. Association between non-alcoholic fatty liver disease and risk of new-onset atrial fibrillation in healthy adults. Liver Int 40, 338-346, doi:10.1111/liv.14236 (2020).

6. To, T. et al. Global asthma prevalence in adults: findings from the cross-sectional world health survey. BMC Public Health 12,204 , doi:10.1186/1471-2458-12-204 (2012).

7. Ahmadizar, F. et al. Childhood obesity in relation to poor asthma control and exacerbation: a meta-analysis. Eur Respir J 48, 10631073, doi:10.1183/13993003.00766-2016 (2016).

8. Beuther, D. A. \& Sutherland, E. R. Overweight, obesity, and incident asthma: a meta-analysis of prospective epidemiologic studies. Am J Respir Crit Care Med 175, 661-666, doi:10.1164/rccm.200611-17170C (2007).

9. Brumpton, B. M. et al. Metabolic syndrome and incidence of asthma in adults: the HUNT study. Eur Respir J 42, 1495-1502, doi:10.1183/09031936.00046013 (2013).

10. Adeyeye, O. O. et al. Understanding asthma and the metabolic syndrome - a Nigerian report. Int Arch Med 5, 20, doi:10.1186/17557682-5-20 (2012).

11. Park, J. et al. Diseases concomitant with asthma in middle-aged and elderly subjects in Korea: a population-based study. Allergy Asthma Immunol Res 5, 16-25, doi:10.4168/aair.2013.5.1.16 (2013).

12. Marchesini, G. et al. Nonalcoholic fatty liver, steatohepatitis, and the metabolic syndrome. Hepatology 37, 917-923, doi:10.1053/jhep.2003.50161 (2003).

13. Bugianesi, E., Moscatiello, S., Ciaravella, M. F. \& Marchesini, G. Insulin resistance in nonalcoholic fatty liver disease. Curr Pharm Des 16, 1941-1951, doi:10.2174/138161210791208875 (2010).

14. Woo, A. et al. Incidence of cancer after asthma development: 2 independent population-based cohort studies. Journal of Allergy and Clinical Immunology, doi:https://doi.org/10.1016/j.jaci.2020.04.041 (2020).

15. Lee, H. et al. Coexisting COPD Increases Mortality in Patients With Corticosteroid-Dependent Asthma: A Nationwide Population-Based Study. Allergy Asthma Immunol Res 12, 821-831 (2020).

16. Park, S.-Y. et al. High Prevalence of Asthma in Elderly Women: Findings From a Korean National Health Database and Adult Asthma Cohort. Allergy Asthma Immunol Res 10, 387-396 (2018).

17. Lee, T. et al. Risk Factors for Asthma-Related Healthcare Use: Longitudinal Analysis Using the NHI Claims Database in a Korean Asthma Cohort. PLOS ONE 9, e112844, doi:10.1371/journal.pone.0112844 (2014).

18. World Health Organization. Regional Office for the Western, P. The Asia-Pacific perspective : redefining obesity and its treatment. 55p. Coordinated by the International Diabetes Institute; co-sponsored jointly by the Regional Office for the Western Pacific (WPRO), World Health Organization, the International Association for the Study of Obesity and the International Obesity Task Force (Sydney : Health Communications Australia, 2000).

19. Huang, X. et al. Validation of the Fatty Liver Index for Nonalcoholic Fatty Liver Disease in Middle-Aged and Elderly Chinese. Medicine (Baltimore) 94, e1682, doi:10.1097/md.0000000000001682 (2015).

20. Yadav, D. et al. Fatty liver index as a simple predictor of incident diabetes from the KoGES-ARIRANG study. Medicine (Baltimore) 95 , e4447, doi:10.1097/md.0000000000004447 (2016).

21. Bedogni, G. et al. The Fatty Liver Index: a simple and accurate predictor of hepatic steatosis in the general population. BMC Gastroentero/ 6, 33, doi:10.1186/1471-230x-6-33 (2006).

22. Yang, B. L. et al. External validation of fatty liver index for identifying ultrasonographic fatty liver in a large-scale cross-sectional study in Taiwan. PLoS One 10, e0120443, doi:10.1371/journal.pone.0120443 (2015).

23. Choi, Y. J. et al. Is nonalcoholic fatty liver disease associated with the development of prostate cancer? A nationwide study with 10,516,985 Korean men. PLoS One 13, e0201308, doi:10.1371/journal.pone.0201308 (2018).

24. Ballestri, S. et al. Nonalcoholic fatty liver disease is associated with an almost twofold increased risk of incident type 2 diabetes and metabolic syndrome. Evidence from a systematic review and meta-analysis. J Gastroenterol Hepato/ 31, 936-944, doi:10.1111/jgh.13264 (2016).

25. Kim, J. Y. et al. Association between Fatty Liver Index and Periodontitis: the Korea National Health and Nutrition Examination Survey. Sci Rep 10, 3805, doi:10.1038/s41598-020-60797-7 (2020).

26. Leone, N. et al. Lung function impairment and metabolic syndrome: the critical role of abdominal obesity. Am J Respir Crit Care Med 179, 509-516, doi:10.1164/rccm.200807-11950C (2009). 
27. Cottrell, L., Neal, W. A., Ice, C., Perez, M. K. \& Piedimonte, G. Metabolic abnormalities in children with asthma. Am J Respir Crit Care Med 183, 441-448, doi:10.1164/rccm.201004-06030C (2011).

28. Unger, R. H. Minireview: weapons of lean body mass destruction: the role of ectopic lipids in the metabolic syndrome. Endocrinology 144, 5159-5165, doi:10.1210/en.2003-0870 (2003).

29. Shi, H. et al. TLR4 links innate immunity and fatty acid-induced insulin resistance. J Clin Invest 116, 3015-3025, doi:10.1172/jci28898 (2006).

30. Hotamisligil, G. S. Endoplasmic reticulum stress and the inflammatory basis of metabolic disease. Cel/ 140, $900-917$, doi:10.1016/j.cell.2010.02.034 (2010).

31. Han, Y. Y., Forno, E. \& Celedón, J. C. Adiposity, fractional exhaled nitric oxide, and asthma in U.S. children. Am J Respir Crit Care Med 190, 32-39, doi:10.1164/rccm.201403-05650C (2014).

32. Scott, H. A., Gibson, P. G., Garg, M. L. \& Wood, L. G. Airway inflammation is augmented by obesity and fatty acids in asthma. Eur Respir J 38, 594-602, doi:10.1183/09031936.00139810 (2011).

33. Dekkers, B. G., Schaafsma, D., Tran, T., Zaagsma, J. \& Meurs, H. Insulin-induced laminin expression promotes a hypercontractile airway smooth muscle phenotype. Am J Respir Cell Mol Biol 41, 494-504, doi:10.1165/rcmb.2008-02510C (2009).

34. Nie, Z., Jacoby, D. B. \& Fryer, A. D. Hyperinsulinemia potentiates airway responsiveness to parasympathetic nerve stimulation in obese rats. Am J Respir Cell Mol Biol 51, 251-261, doi:10.1165/rcmb.2013-04520C (2014).

35. Kuschnir, F. C. et al. Severe asthma is associated with metabolic syndrome in Brazilian adolescents. J Allergy Clin Immuno/ 141, 19471949.e1944, doi:10.1016/j.jaci.2018.01.026 (2018).

36. Wang, L. et al. Sex difference in the association between obesity and asthma in U.S. adults: Findings from a national study. Respir Med 109, 955-962, doi:10.1016/j.rmed.2015.06.001 (2015).

37. Kim, S. \& Camargo, C. A., Jr. Sex-race differences in the relationship between obesity and asthma: the behavioral risk factor surveillance system, 2000. Ann Epidemiol 13, 666-673, doi:10.1016/s1047-2797(03)00054-1 (2003).

38. Huovinen, E., Kaprio, J. \& Koskenvuo, M. Factors associated to lifestyle and risk of adult onset asthma. Respir Med $\mathbf{9 7 , 2 7 3 - 2 8 0 ,}$ doi:10.1053/rmed.2003.1419 (2003).

39. Park, S., Choi, N. K., Kim, S. \& Lee, C. H. The relationship between metabolic syndrome and asthma in the elderly. Sci Rep 8, 9378 , doi:10.1038/s41598-018-26621-z (2018).

40. Varraso, R., Siroux, V., Maccario, J., Pin, I. \& Kauffmann, F. Asthma severity is associated with body mass index and early menarche in women. Am J Respir Crit Care Med 171, 334-339, doi:10.1164/rccm.200405-6740C (2005).

41. Luder, E., Ehrlich, R. I., Lou, W. Y., Melnik, T. A. \& Kattan, M. Body mass index and the risk of asthma in adults. Respir Med 98 , 29-37, doi:10.1016/j.rmed.2003.08.004 (2004).

42. Shore, S. A. \& Johnston, R. A. Obesity and asthma. Pharmacol Ther 110, 83-102, doi:10.1016/j.pharmthera.2005.10.002 (2006).

43. Beuther, D. A. Obesity and asthma. Clin Chest Med 30, 479-488, viii, doi:10.1016/j.ccm.2009.05.002 (2009).

44. Leoni, S. et al. Current guidelines for the management of non-alcoholic fatty liver disease: A systematic review with comparative analysis. World J Gastroenterol 24, 3361-3373, doi:10.3748/wjg.v24.i30.3361 (2018).

45. Shin, J.-Y. et al. Changing patterns of adult asthma incidence: results from the National Health Insurance Service-National Sample Cohort (NHIS-NSC) database in Korea. Sci Rep 8, 15052-15052, doi:10.1038/s41598-018-33316-y (2018).

46. Calori, G. et al. Fatty liver index and mortality: the Cremona study in the 15th year of follow-up. Hepatology 54, 145-152, doi:10.1002/hep.24356 (2011).

\section{Tables}

Table 1. Baseline Characteristics of study population 


\begin{tabular}{|c|c|c|c|c|}
\hline \multirow{3}{*}{ Variables } & \multicolumn{4}{|c|}{ Fatty liver index (FLI) } \\
\hline & $0 \leq \mathrm{FLI}<30$ & $30 \leq \mathrm{FLI}<60$ & $F L I \geq 60$ & $P$ value \\
\hline & $N=136,094$ & $N=18,373$ & $N=6,136$ & \\
\hline Age & $40.7 \pm 12.3$ & $45.4 \pm 12.3$ & $42.6 \pm 11.6$ & $<0.001$ \\
\hline Sex (male, \%) & $2,574(18.8)$ & $9,462(51.5)$ & $4,062(66.2)$ & $<0.001$ \\
\hline $\mathrm{BMI}\left(\mathrm{Kg} / \mathrm{m}^{2}\right)$ & $21.9 \pm 2.5$ & $26.1 \pm 2.4$ & $28.8 \pm 3.5$ & $<0.001$ \\
\hline WC (cm) & $73.1 \pm 7.1$ & $85.6 \pm 5.6$ & $92.5 \pm 7.2$ & $<0.001$ \\
\hline SBP, mmHg & $114.0 \pm 11.4$ & $120.7 \pm 10.4$ & $123.2 \pm 9.7$ & $<0.001$ \\
\hline DBP, mmHg & $71.1 \pm 8.1$ & $75.3 \pm 7.4$ & $77.2 \pm 6.9$ & $<0.001$ \\
\hline Alcohol consumption (g/week) & $26.6 \pm 65.8$ & $48.1 \pm 105.6$ & $76.5 \pm 149.6$ & $<0.001$ \\
\hline \multicolumn{5}{|l|}{ (met-min/week) } \\
\hline \multicolumn{5}{|l|}{ Laboratory data } \\
\hline Fasting glucose, mg/dL & $89.8 \pm 10.0$ & $94.0 \pm 11.1$ & $96.1 \pm 11.6$ & $<0.001$ \\
\hline Total cholesterol, mg/dL & $186.9 \pm 35.8$ & $206.0 \pm 45.9$ & $214.0 \pm 47.4$ & $<0.001$ \\
\hline LDL cholesterol, mg/dL & $113.6 \pm 168.5$ & $125.5 \pm 97.5$ & $123.9 \pm 80.7$ & $<0.001$ \\
\hline HDL cholesterol, mg/dL & $61.0 \pm 23.1$ & $53.7 \pm 37.2$ & $52.8+43.7$ & $<0.001$ \\
\hline Triglyceride, mg/dL & $85.8 \pm 43.7$ & $164.9 \pm 90.8$ & $238.2 \pm 193.3$ & $<0.001$ \\
\hline GGT, U/L & $17.8 \pm 11.3$ & $38.4 \pm 33.1$ & $71.4 \pm 72.4$ & $<0.001$ \\
\hline
\end{tabular}

$\mathrm{BMI}$ = body mass index; DBP = diastolic blood pressure; FLI = fatty liver index; GGT = y-glutamyltransferase; $\mathrm{HDL}=$ high-density lipoprotein; $\mathrm{LDL}=$ low-density lipoprotein; SBP= systolic blood pressure; WC = waist circumference.

Table 2. Association between fatty liver index and incidence of adult-onset asthma 


\begin{tabular}{|c|c|c|c|c|c|c|c|c|c|c|c|}
\hline & & & Univariate & & & Model $1^{*}$ & & & Model $2^{\dagger}$ & & \\
\hline & $\begin{array}{l}\text { Total } \\
(\mathrm{N})\end{array}$ & Event $(n, \%)$ & $\mathrm{HR}$ & $\begin{array}{l}95 \% \\
\mathrm{Cl}\end{array}$ & $\begin{array}{l}P \\
\text { value }\end{array}$ & $\mathrm{HR}$ & $\begin{array}{l}(95 \% \\
\mathrm{Cl})\end{array}$ & $\begin{array}{l}P \\
\text { value }\end{array}$ & $\mathrm{HR}$ & $\begin{array}{l}95 \% \\
\mathrm{Cl}\end{array}$ & $\begin{array}{l}P \\
\text { value }\end{array}$ \\
\hline \multicolumn{12}{|l|}{ FLI group } \\
\hline $\begin{array}{l}0 \leq \mathrm{FLI}< \\
30\end{array}$ & 136,094 & $13,744(10.1)$ & Reference & & & Reference & & & Reference & & \\
\hline $\begin{array}{l}30 \leq \mathrm{FLI} \\
<60\end{array}$ & 18,373 & 1,986 (10.8) & 1.04 & $\begin{array}{l}0.99- \\
1.09\end{array}$ & 0.06 & 1.13 & $\begin{array}{l}1.07- \\
1.18\end{array}$ & $<0.001$ & 1.14 & $\begin{array}{l}1.08- \\
1.20\end{array}$ & $<0.001$ \\
\hline $\mathrm{FLI} \geq 60$ & 6136 & 647 (10.5) & 1.04 & $\begin{array}{l}0.96- \\
1.12\end{array}$ & 0.28 & 1.23 & $\begin{array}{l}1.13- \\
1.33\end{array}$ & $<0.001$ & 1.25 & $\begin{array}{l}1.15- \\
1.36\end{array}$ & $<0.001$ \\
\hline \multicolumn{12}{|l|}{ FLI criteria ${ }^{\ddagger}$} \\
\hline $\begin{array}{l}\text { low } \\
\text { likelihood }\end{array}$ & 103630 & $9846(10.5)$ & Reference & & & Reference & & & Reference & & \\
\hline $\begin{array}{l}\text { intermediate } \\
\text { likelihood }\end{array}$ & 25797 & $2943(11.4)$ & 1.11 & $\begin{array}{l}1.06- \\
1.14\end{array}$ & $\dot{0} 001$ & 1.09 & $\begin{array}{l}1.05- \\
1.13\end{array}$ & 0.001 & 1.10 & $\begin{array}{l}1.06- \\
1.14\end{array}$ & $\dot{0} 001$ \\
\hline $\begin{array}{l}\text { High } \\
\text { likelihood }\end{array}$ & 31176 & $3588(11.5)$ & 1.12 & $\begin{array}{l}1.08- \\
1.15\end{array}$ & $<.001$ & 1.17 & $\begin{array}{l}1.13- \\
1.19\end{array}$ & $<.001$ & 1.18 & $\begin{array}{l}1.14- \\
1.22\end{array}$ & $<.001$ \\
\hline \multicolumn{12}{|l|}{ HSI } \\
\hline $\mathrm{HIS}<36$ & 139608 & 14044(10.1) & Reference & & & Reference & & & Reference & & \\
\hline $\mathrm{HIS} \geq 36$ & 20995 & $2333(11.1)$ & 1.10 & $\begin{array}{l}1.06- \\
1.15\end{array}$ & $\hat{0}_{0.001}$ & 1.09 & $\begin{array}{l}1.04- \\
1.14\end{array}$ & $<0.001$ & 1.12 & $\begin{array}{l}1.07- \\
1.17\end{array}$ & $<0.001$ \\
\hline
\end{tabular}

${ }^{*}$ Cox proportional hazard models including age, and sex as covariates

${ }^{\dagger}$ Cox proportional hazard models including Model 1 plus activity, drinking, systolic blood pressure, diastolic blood pressure, fast blood glucose and low density lipoprotein cholesterol as covariates

‡Low likelihood: $0 \leq \mathrm{FLI}<25$ for male, $0 \leq \mathrm{FLI}<10$ for female; intermediate likelihood: $25 \leq \mathrm{FLI}<35$ for male, $10 \leq \mathrm{FLI}<20$ for female; high likelihood: $\mathrm{FLI} \geq 35$ for male, $\mathrm{FLI} \geq 20$ for female

$\mathrm{Cl}=$ confidence interval; $\mathrm{FLI}=$ fatty liver index; $\mathrm{HR}$ = hazard ratio; $\mathrm{HIS}=$ Hepatic steatosis index

Table 3. sex specific associations between fatty liver index and incidence of asthma 


\begin{tabular}{|c|c|c|c|c|c|c|c|c|c|c|c|}
\hline & & & Univariate & & & Model $1^{*}$ & & & Model $2^{\dagger}$ & & \\
\hline & $\begin{array}{l}\text { Total } \\
(\mathrm{N})\end{array}$ & Event (n, \%) & $\mathrm{HR}$ & $95 \% \mathrm{Cl}$ & $\begin{array}{l}P \\
\text { value }\end{array}$ & $\mathrm{HR}$ & $\begin{array}{l}(95 \% \\
\mathrm{Cl})\end{array}$ & $\begin{array}{l}P \\
\text { value }\end{array}$ & $\mathrm{HR}$ & $\begin{array}{l}95 \% \\
\mathrm{Cl}\end{array}$ & $\begin{array}{l}P \\
\text { value }\end{array}$ \\
\hline \multicolumn{12}{|l|}{ Female } \\
\hline $\begin{array}{l}0 \leq \mathrm{FLI} \\
<30\end{array}$ & 110522 & $11794(10.7)$ & Reference & & & Reference & & & Reference & & \\
\hline $\begin{array}{l}30 \leq \mathrm{FLI} \\
<60\end{array}$ & 8919 & $1175(13.2)$ & 1.23 & $\begin{array}{l}1.16- \\
1.31\end{array}$ & $<0.001$ & 1.17 & $\begin{array}{l}1.10- \\
1.24\end{array}$ & $<0.001$ & 1.18 & $\begin{array}{l}1.11- \\
1.26\end{array}$ & $<0.001$ \\
\hline $\mathrm{FLI} \geq 60$ & 2071 & $313(15.1)$ & 1.49 & $\begin{array}{l}1.33- \\
1.66\end{array}$ & $<0.001$ & 1.44 & $\begin{array}{l}1.28- \\
1.61\end{array}$ & $<0.001$ & 1.46 & $\begin{array}{l}1.13- \\
1.64\end{array}$ & $<0.001$ \\
\hline \multicolumn{12}{|l|}{ Male } \\
\hline $\begin{array}{l}0 \leq \mathrm{FLI} \\
<30\end{array}$ & 25572 & 1950(7.6) & Reference & & & Reference & & & Reference & & \\
\hline $\begin{array}{l}30 \leq \mathrm{FLI} \\
<60\end{array}$ & 9454 & $811(8.6)$ & 1.09 & $\begin{array}{l}1.01- \\
1.18\end{array}$ & 0.03 & 1.048 & $\begin{array}{l}0.96- \\
1.13\end{array}$ & 0.25 & 1.05 & $\begin{array}{l}0.97- \\
1.15\end{array}$ & 0.182 \\
\hline $\mathrm{FLI} \geq 60$ & 4065 & $334(8.2)$ & 1.07 & $\begin{array}{l}0.95- \\
1.2\end{array}$ & 0.23 & 1.049 & $\begin{array}{l}0.93- \\
1.17\end{array}$ & 0.42 & 1.07 & $\begin{array}{l}0.94- \\
1.20\end{array}$ & 0.275 \\
\hline
\end{tabular}

${ }^{*}$ Cox proportional hazard models including age as covariates

${ }^{\dagger}$ Cox proportional hazard models including Model 1 plus activity, BMI, drinking, systolic blood pressure, diastolic blood pressure, fast blood glucose and low density lipoprotein cholesterol as covariates

$\mathrm{Cl}=$ confidence interval; $\mathrm{FLI}=$ fatty liver index; $\mathrm{HR}=$ hazard ratio

\section{Table 4. Asthma-related healthcare use between FLI groups}




\begin{tabular}{|c|c|c|c|c|}
\hline Variables & $0 \leq \mathrm{FLI}<30$ & $30 \leq \mathrm{FLI}<60$ & $\mathrm{FLI} \geq 60$ & $P$ values \\
\hline $\mathrm{N}(\%)^{*}$ & 13744(83.9) & 1986(12.1) & $647(4.0)$ & \\
\hline Hospitalizations & & & & $<0.001$ \\
\hline Never & $9155(66.5 \%)$ & $1287(64.9 \%)$ & $456(70.5 \%)$ & \\
\hline Ever $^{\dagger}$ & $4589(33.5 \%)$ & $699(35.1 \%)$ & $191(29.5 \%)$ & \\
\hline Mean $\pm S D^{\ddagger}$ & $2.08 \pm 2.97$ & $2.14 \pm 3.08$ & $2.54 \pm 3.16$ & $<0.001$ \\
\hline \multicolumn{5}{|l|}{ Hospitalization days $\S$} \\
\hline $\begin{array}{l}\text { per capita } \\
\qquad(\text { Mean, SD) }\end{array}$ & $8.18 \pm 15.19$ & $14.02 \pm 20.11$ & $19.78 \pm 21.25$ & $<0.001$ \\
\hline $\begin{array}{l}\text { per each admission } \\
\text { (Mean, SD) }\end{array}$ & $8.72 \pm 8.11$ & $9.34 \pm 10.99$ & $11.54 \pm 8.38$ & $<0.001$ \\
\hline ICU hospitalizations & & & & $<0.001$ \\
\hline Never & 13685(99.96\%) & 192(99.93\%) & $642(99.92 \%)$ & \\
\hline Ever & $59(0.04 \%)$ & $14(0.07 \%)$ & $5(0.08 \%)$ & \\
\hline Mean \pm SD & $1.21 \pm 0.51$ & $1.22 \pm 0.24$ & $1.27 \pm 0.71$ & 0.283 \\
\hline \multicolumn{5}{|l|}{ ED visit" } \\
\hline Never & 13744 & 1986 & 647 & \\
\hline Ever & - & - & - & \\
\hline Mean \pm SD & - & - & - & \\
\hline No. of outpatient visits & & & & $<0.001$ \\
\hline Never & $5523(40.3 \%)$ & $750(37.9 \%)$ & $242(36.6 \%)$ & \\
\hline Ever & $8211(59.7 \%)$ & $1231(62.1 \%)$ & $420(63.4 \%)$ & \\
\hline Mean \pm SD & $3.42 \pm 4.87$ & $3.45 \pm 7.91$ & $3.64 \pm 8.2$ & 0.068 \\
\hline
\end{tabular}

*Total number of new onset asthma in each FLI group

${ }^{\dagger}$ Number of patients who underwent asthma-related hospitalization at least once.

${ }^{\ddagger}$ Average number of admissions per person among patients who had asthma-related hospitalizations at least once.

$\S$ Average length of stay among patients who had asthma-related hospitalizations at least once (per person or per admission).

'Included patients who underwent asthma-related ED visits without admission

$\mathrm{ED}=$ Emergency department; $\mathrm{ICU}=$ Intensive care unit

\section{Figures}


- 2,607 who were under the age of 20

- 70,315 diagnosed as asthma within 2 years before the index check-up

- 156,575 diagnosed to have comorbid conditions within 2 year before the index check-up

(liver disease, congestive heart failure, hypertension, diabetes, cerebrovascular disease, ischemic heart disease, peripheral vascular disease, other pulmonary disease, valvular heart disease)

- 15,457 who were prescribed oral hypoglycemic, antihypertensive, or lipid lowering agent within 1 year before the index check-up

- 128,275 who answered that they have smoked or smoked in the past

- 2,160 who answered questionnaire to have comorbid conditions at the index check-up (cerebrovascular disease, heart disease, hypertension, and diabetes)

- 14,618 whose systolic or diastolic blood pressures measured $\geq 140$ or $\geq 90$, respectively, at the index check-up

- 2,552 whose fasting glucose measured $\geq 126$ at the index check-up

- 3,722 whose data for the index check-up were missing

\section{Figure 1}

Overview of the study population

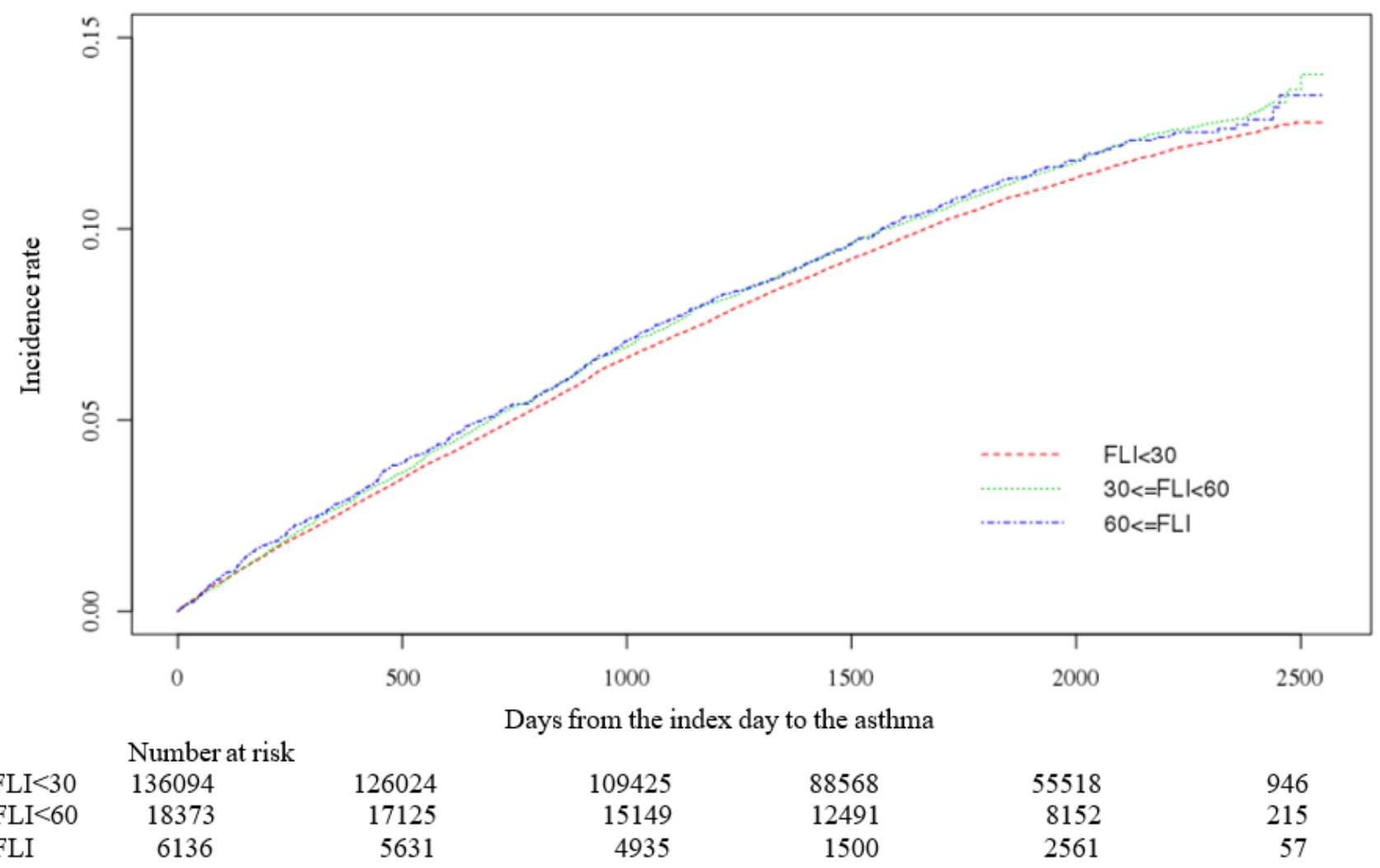




\section{Figure 2}

Cumulative incidence of adult-onset asthma by FLI groups

\section{Supplementary Files}

This is a list of supplementary files associated with this preprint. Click to download.

- 20210601AsthmaandFLIsupplementaryFinal.docx 